\title{
Design and Evaluation of Interaction Technology for Medical Team Meetings
}

\author{
Alex Olwal, Oscar Frykholm, Kristina Groth, and Jonas Moll \\ School of Computer Science and Communication \\ KTH (Royal Institute of Technology), Stockholm, Sweden \\ \{alx, frykholm,kicki, jomol\}@esc.kth.se
}

\begin{abstract}
Multi-disciplinary team meetings (MDTMs) are essential in healthcare, where medical specialists discuss diagnosis and treatment of patients. We introduce a prototype multi-display groupware system, intended to augment the discussions of medical imagery, through a range of input mechanisms, multiuser interfaces and interaction techniques on multi-touch devices and pen-based technologies. Observations of MDTMs, as well as interviews and observations of surgeons and radiologists, serve as a foundation for guidelines and a set of implemented techniques. We present a detailed analysis of a study where the techniques' potential was explored with radiologists and surgeons of different specialties and varying expertise. The results show that the implemented technologies have the potential to bring numerous benefits to the team meetings with minimal modification to the current workflow. We discuss how they can augment the expressiveness and communication between meeting participants, facilitate understanding for novices, and improve remote collaboration.
\end{abstract}

Keywords: Medical team meetings, collaboration, single-display groupware, multi-display groupware, multi-touch, pen, mobile.

\section{Introduction}

Multi-disciplinary teams in modern healthcare are facilitating the discussion of patients, decisions on diagnosis and treatment, operation planning, interventions and surgery $[5,7,9,15,10,20,6]$. The medical specialists participating in the meetings are not always located in the same facilities, or even city, whereby tele- or videoconferencing is frequently used. During the meetings, specialists present relevant information about patient cases, based on their domain-specific expertise. Current meeting facilities typically only allow image presentation and demonstration by the radiologist and/or pathologist, whereas the other meeting participants have no means for interacting with the material $[7,9,11,5]$. To support the changing style of collaboration, new, effective user interfaces are needed.

We have worked closely with a surgical department at a university hospital, which focuses on complicated deceases in the upper abdomen, where multi-disciplinary team meetings (MDTMs) are a central part of patient care. In this context, we are interested in applying and evaluating technology that can improve understanding, 

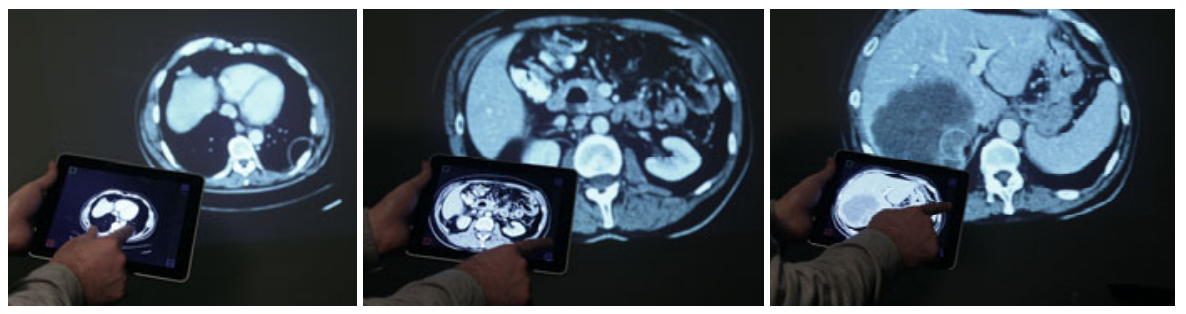

Fig. 1. Synchronized interaction with projected radiology material, using multi-touch gestures and widgets to zoom and scroll through the image stack

simplify group discussion and increase collaboration for safer and faster medical decisions [5]. We have therefore developed a set of tools and techniques, focusing on multi-display groupware (MDG), as shown in Figure 1. In the spirit of previous work $[1,13,16,19,21]$, our goal has been to distribute the interactive capabilities at such meetings among participants. While our work is applied to the medical domain, we also find it generally applicable to other types of MDG and single-display groupware (SDG) scenarios $[21,13,23]$. To ensure qualitative feedback without influence from peers and to minimize the effect of time constraints, this study explores the collaborative techniques with one participant at a time in a simulated team meeting. This first evaluation of tools for facilitated collaboration in MDTMs will form the basis for a large-scale user testing in the real setting.

We first review related work, followed by an overview of the medical team meetings that we are studying and a discussion of a number of initial studies that we conducted, which form the basis for this work. We then present our method, a set of design guidelines and the implemented techniques. The main part focuses on our user study and results, followed by a Discussion, Conclusions and Future Work.

\section{Background}

Several researchers report on detailed field studies of MDTMs [e.g., 5, 6, 7, 8, 9, 11]. Time is an important aspect when judging the efficiency of the meeting $[7,5]$ and supporting technology must take this into account, as well as specific participant roles [6]. Although some problems regarding efficiency and effects on the group work process were likely to arise, Kane and Luz [7] acknowledge the need for technology for collaborative decision-making. Unsupported needs include the ability to point to areas of interest and to make annotations on shared displays [8]. Laser pointers were shown to partially alleviate these deficiencies, although it was noted that the laser dot could not be seen at remote locations during teleconferencing meetings [7].

Several projects have explored digital tools for multi-user interaction on single or multiple displays. The Pebbles Project $[13,14]$ is a collaboration framework for multiple handheld computers that are connected to a shared display, providing remote keyboard and mouse control, and multi-user drawing with individual cursors. M-Pad [19] uses tracked handhelds for advanced multi-user interaction techniques with whiteboards through toolglasses, palettes, cross-device transfer, and annotations. While these systems were pioneering, they had no wireless connectivity and their graphics performance limited possibilities for manipulating medical images. 

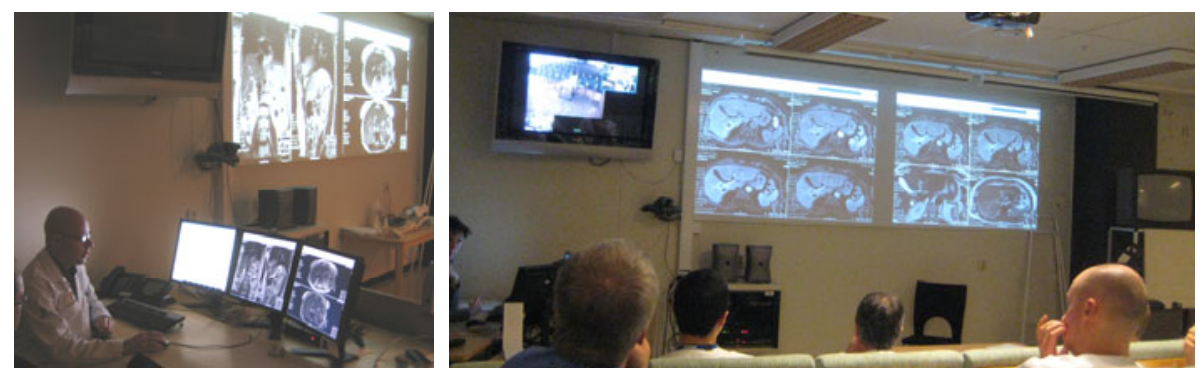

Fig. 2. Left) Radiologist controls the interaction with the imagery from his workstation by navigating (scrolling through image stacks) and pointing out findings with the mouse. Right) Medical specialists from different disciplines (e.g., pathology, oncology and hepatology) view and discuss the imagery from a distance, and can only refer to areas of interest through verbal means or finger pointing.

Architectures for mobile interaction with shared displays [16] and multi-cursor interaction [1] illustrate the complexity when designing multi-display groupware environments. Tse et al. [22] describe a set of experiments where shared workspaces were partitioned by participants into individual areas to address interference, while one of the motivations for designing cooperative gestures [12] was to clarify users' intentions. Wallace et al. [23] discuss the results from a study comparing SDGs (multiple input devices, single display) with MDGs (multiple input devices, individual displays). They conclude that SDGs benefit in awareness of collaborators' activities, but may be distracting and can interfere with the group's primary tasks. MDGs, on the other hand, may provide a private, customizable, workspace with fewer distractions, but can instead require more efforts for coordinating team work.

Lee et al. [10] and Eng et al. [2] describe remote consultations where radiologists and physicians use a synchronized image viewer. The systems enable shared reviewing with joint image navigation, synchronized cursors for pointing, and image annotations. Shareable displays [18] have also been developed using PDAs to access and navigate radiology material on larger displays. Recent advances in mobile technology, allow us to overcome the limitations of previous systems [3] to support distributed interaction with radiology imagery.

Our paper builds on a body of work, in which we have studied a large number of MDTMs at a gastro-surgical department over four years. Results from these studies indicate many benefits if also participants in these meetings could navigate the material, point to regions of interest, and annotate important features.

\subsection{MDTMs at Gastro}

The centralization of specialized medical care to regional university hospitals is a recent strategy for quality improvement and effectiveness. The gastro-surgical department (Gastro) that we have studied has the regional responsibility for specialised care in the upper part of the abdomen (i.e., complex diseases in the liver, pancreas and esophagus). The 25 surgeons collaborate closely with several other units within the university hospital organisation, including pathology, radiology, oncology, 
and hepatology. The weekly MDTMs (See Figure 2) are an important element of the work process at Gastro.

The patient care pathway at Gastro [4] consists of four main steps: coordination of medical material, decision on diagnosis and treatment, surgical treatment, and postoperative treatment. Gastro regularly uses three types of MDTMs during the patient care pathway.

The Decision Meeting is a forum for discussion of patient diagnosis, operability and resectability, to decide on the next steps in the care, such as surgery, radiology, oncology treatment, or the need for additional examinations. There are a number of key roles at the meeting. First, a surgeon summarizes the patient's medical history and formalises the discussion topic. Second, a radiologist, seated at a workstation with three high-resolution displays, presents the radiological analysis, while participants follow the walk-through of the medical imagery on two projection screens. Third, if there are pathology reports, then these are presented by a pathologist. Fourth, a senior surgeon leads the following discussion, which relies on the display and navigation of medical imagery, to reach a consensus decision. A group of medical specialists from different disciplines participate with comments, questions and discussions. Less experienced medical doctors attend as part of their training, but do not actively participate in the discussions. The meetings must be streamlined and efficient due to a high number of patients to discuss by the many attendees under time constraints.

Pre-operative Meetings (Pre-ops) are similar to Decision Meetings, but have fewer participants, are more focused on operation strategy and planning and have no time constraints. They are typically attended by three to six surgeons, one radiologist and occasionally one pathologist, all from the same hospital, and take place the week before surgery. The video link is not used, but specialists from other departments can participate if needed.

Post-operative Meetings (Post-ops) allow pre-op attendees to share their experiences after surgery to, for example, discuss how well the pre-op planning matched the surgical procedures. These are only conducted for pancreas cases.

\subsection{Initial Studies at Gastro}

Our work is based on four years of field studies where data has been collected in an on-going process of ethnographic fieldwork and participatory design projects. Our early MDTM observations have showed a need, but limited possibilities, for interaction with patient information. To better understand the interaction with radiology imagery during MDTMs, we observed and analysed twelve additional Decision Meetings and Pre-ops during 2009. The meetings were video recorded and the sections where surgeons specifically tried to point to and navigate in the images were analysed in detail. Similar to related work [15], it was observed that the radiologist relies heavily on mouse pointing when presenting and explaining the medical imagery. The analysis of the observations show that also the surgeons point to parts in the medical imagery (from their seats or by approaching the screen) to illustrate how operations should be performed or when asking for clarifications. This indicates a need to illustrate a procedure or make a deictic reference to a detail in the imagery. It was also clear that the radiologist, surgeons and other specialists have a formalized language with an established common ground that make it easier to 
verbally refer to parts in the imagery during their discussions. We also observed occasions where some participants used laser pointers to clarify their intentions.

To further understand the interaction during MDTMs, a follow-up field study of three consecutive Pre-ops (each lasting 40 min) was conducted in which laser pointers were handed out to all participants, except the radiologist. The objective was to observe how laser pointer usage would affect meeting discussions. Participants were not given specific instructions on how or when to use the laser pointers. In the analysis, we found that the laser pointers were used:

1) To point and "draw" in different parts of the projected imagery

2) For joint navigation and guidance

3) To help coordinate communication by pointing to smaller features while asking for more detailed information

4) For gestures that allowed surgeons to illustrate plans, e.g., for cuts of organs

The setup's major drawback was that the radiologist had to direct his attention from the workstation to see the laser dot on the projection screens, preventing simultaneous control of the workstation and maintained awareness of the surgeons' pointing.

The advantage of laser pointers, from a technological standpoint, is low cost, portability and infrastructural independence. Still, despite the benefits and immediate availability, laser pointers have not been widely adopted by participants at the MDTMs. From observations it became clear that laser pointers do not meet the requirements of multi-display meetings, where radiologists, surgeons, and remote participants are viewing three separate, but digitally synchronized displays. A surgeon's laser pointer on the projection will thus not be visible on the radiologist's workstation or through the videoconferencing system, which turns out to be prohibitive for practical use. Also, the laser pointer study showed that the person pointing is using words like "here" instead of verbally explaining a part in the image, which is making the referencing even more ambiguous for participants viewing other displays. While it would be possible to track and distribute laser dots as digital cursors using cameras [17], the required infrastructural modifications inspired us to instead develop techniques that would support more advanced and flexible multi-user interaction through digital tools, without the need to install any hardware in the MDTM room.

\section{Prototypes for Collaborative Interaction Techniques}

The objective of the study was to explore the functionality that new interaction techniques and devices can enable for MDTMs, and evaluate their potential for improving understanding, group discussion, collaboration and decision making. Data from our initial studies allowed us to make informed design decisions for the candidate interaction technology to be used in the study. We first decided to prioritize the most important subset of medical imaging functionality from the radiologist's workstation, based on the observed needs of the meeting participants in our studies:

1) Shared/collaborative pointing

2) Navigation (zooming/panning/scrolling in image stack)

3) Annotation (sketching/drawing) 

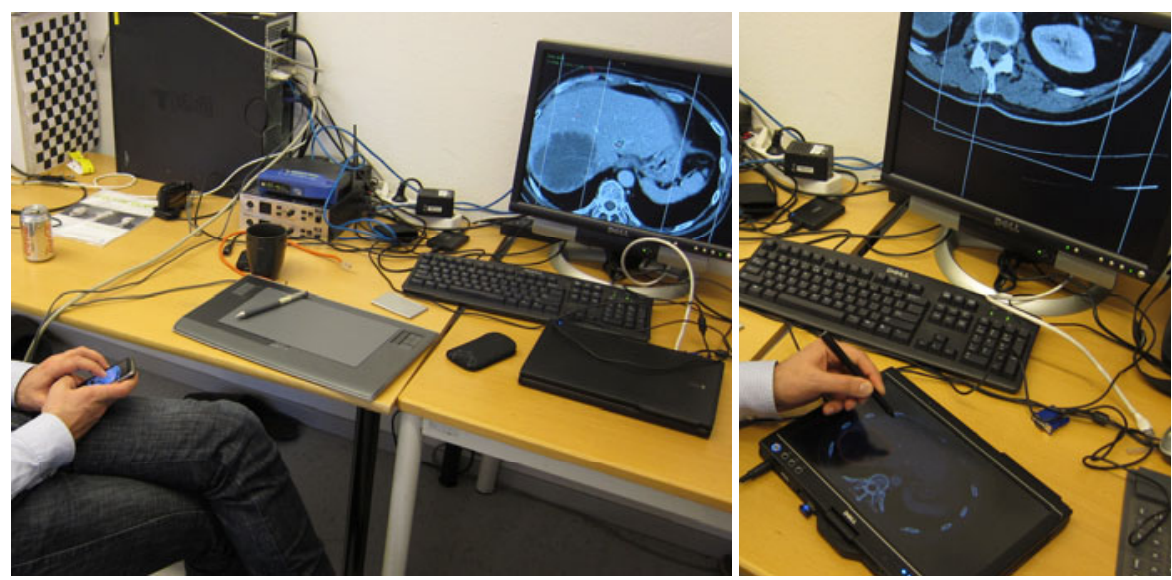

Fig. 3. Surgeons and radiologists participated in interactive prototyping sessions. Left) A radiologist manipulates content on a shared display using a mobile device. Right) Interaction on a Tablet PC (Dell XT2) that supports both pen and multi-touch input.

We interviewed and observed two senior radiologists at their hospital workstations (each $\sim 2 \mathrm{~h}$ ) to gain insight into workflow, important functionality, frequent operations and desired features. We then conducted an interactive workshop with a senior pancreas surgeon $(\sim 3 \mathrm{~h})$ where we discussed the use of digital devices for interacting with the radiology images and related these ideas to typical MDTM discussions. A first version of the prototypes was developed after these sessions. The prototypes were demonstrated and discussed with the surgeon at a second workshop (also $\sim 3 \mathrm{~h}$ ), which provided additional feedback. Two interactive prototyping sessions (each $\sim 2 \mathrm{~h}$ ) were then conducted individually with one of the previously observed radiologists and one new radiologist. Similarly to the second workshop with the surgeon, they individually explored the first version of the prototype interfaces, but also answered questions regarding current practices/problems, potential for the proposed techniques and suggested functionality and features (See Figure 3). Audio and video was recorded for the sessions with the surgeons, and informal notes were taken during the radiologist sessions.

Insights from these sessions supported our preliminary ideas, inspired topics for the user study questionnaire and indicated an interesting potential for collaborative interaction techniques in MDTMs.

\subsection{Design Guidelines}

A set of design guidelines for the tools that we were developing for Pre-ops was identified from our initial studies and prototyping sessions:

Minimal changes to current meeting situation: The physicians are in general satisfied with routines and configuration of current meetings, and that the use of technology is limited to the radiology workstation. We thus wanted our tools to augment the meetings with minimal modifications to current workflow. 
Sporadic interaction with minimal learning time: The users should be able to quickly access the needed functionality, as the dynamic meetings cannot afford complex and time-demanding user interfaces.

Arbitrary number of participants: The system should be able to support interaction from any user through an inherently scalable infrastructure.

Radiologist delegates control: As the radiologist is an expert of interpreting and navigating the medical imagery, and is in control of these processes today, it seems important to maintain that role. While many surgeons will likely want to interact with the imagery, this is not their area of expertise, and may not necessarily be effective. The interviews indicate that distributed interaction could be adopted as long as the radiologist could delegate control when needed.

Synchronization across displays: Current methods (e.g., finger pointing, walking up to the projection screens and the use of laser pointers) exclude remote participants and the radiologist. Digital tools, on the other hand, make it possible to synchronize interaction across multiple displays, which could especially be used to empower remote participants.

\subsection{Interaction Techniques and Implementation}

The design guidelines informed the choice of the technology and architecture for the system. Thus, our system, for example, supports a radiologist's delegation of control, remote participation, and many other features. It is based on a PC server that projects medical imagery, while connected mobile devices are synchronized using the network, such that multiple users can interact using their own device.

Software architecture: Our framework is implemented using $\mathrm{C}++$ and openFrameworks, which allows us to support most of the implemented functionality across multiple platforms (Linux, Mac OS X, Microsoft Windows XP-7 and Apple iOS). The mobile devices are synchronized with the server using OSC (OpenSoundControl), a UDP-based protocol for efficient, platform-independent data streaming.

Pen-based devices: We implemented our techniques on Wacom tablets (no display) and Tablet PCs (Dell XT2) that use electromagnetic pens with high precision/pressure and physical buttons, as shown in Figure 4.
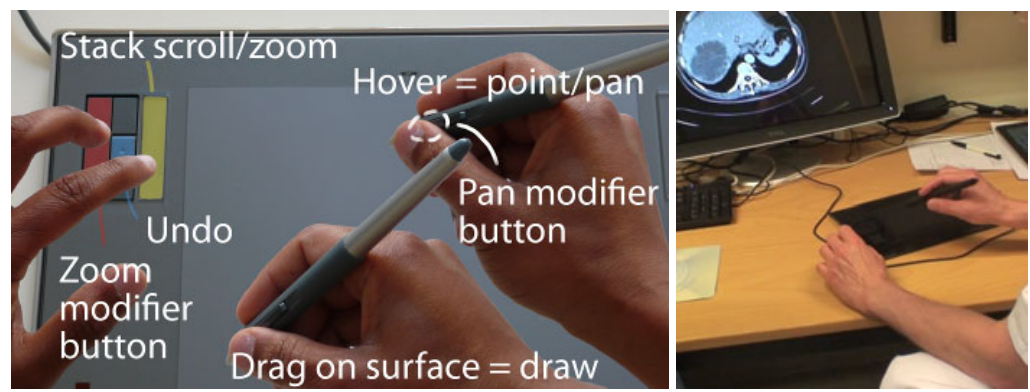

Fig. 4. The tablet uses buttons and a touch strip with a tracked pen for panning, zooming and drawing 

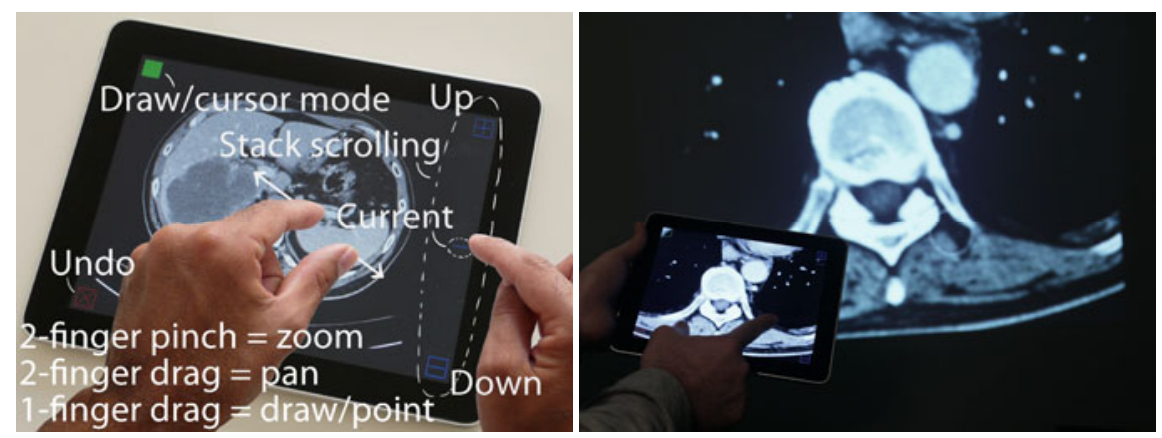

Fig. 5. The multi-touch displays use on-screen buttons and widgets in combination with multitouch gestures

Multi-touch devices: We use Apple iOS devices (3.5" iPod Touch $3^{\text {rd }}$ Generation and 9.7" iPad) as portable multi-touch displays (See Figure 5), and support larger screens through the multi-touch capabilities in Windows 7 (e.g., 21.5" Dell SX2210T, 22" 3M M2256PW, and 12" Dell XT2).

All device types were explored in the prototyping sessions with the surgeon and radiologists, and based on our observations and their feedback we chose to narrow it down to three devices for the user study: the screen-less tablet, and the small and large multi-touch devices (iPod Touch and iPad). We carefully selected the techniques through our initial prototyping and user observations, based on observed gestures at MDTMs and other studies. The hardware was chosen based on commercial availability, mobility, the possibility to use it outside MDTMs, and the different form factors. The pen-based device was selected as the lack of display avoids problem with private focus.

We developed three general interaction techniques for multi-user interaction.

Pointing: One of the primary motivations of the system is to enable participants to point accurately from any location in the room, or over the video conferencing link. It is achieved by touching and dragging with a single finger on the touch-screen devices, or by hovering the pen over the stylus-driven tablet. A coloured cursor distinguishes different users.

Navigation: A central component of the radiology material is composed of CT (computed tomography) or MRI (magnetic resonance imaging) image stacks, where the $3 \mathrm{D}$ volume of the human body and organs are viewed one $2 \mathrm{D}$ slice at a time. Here, zooming, panning and scrolling up/down in the stack are the most important means for navigation. Pan is controlled by dragging two fingers in the multi-touch interfaces, and zoom level is adjusted with two-finger pinching. A widget on the right-hand side visualizes the stack, with absolute access to each slice, and two buttons allow stepping up/down one slice at a time. On the pen-based device, the user pans by pressing a side button on the pen and hovering it over the tablet surface. Stack scrolling is controlled by sliding with a finger of the non-dominant hand on the tablet's touch-sensitive strip, while the simultaneous pressing of a device button zooms instead. 
Annotation: Annotation with individual colours on each slice was implemented to support the exploration of temporary or permanent freehand annotations during the meetings. The multi-touch interface uses an on-screen button to toggle between pointing and drawing, whereas another button allows the user to undo the last stroke for the current slice. Dragging the pen on the tablet surface creates annotations that the user can undo using a dedicated button.

\section{User Study Setup}

We designed a formal qualitative user study to evaluate our interfaces and explore our target group's attitudes towards using digital tools for distributed pointing, navigation and annotation during MDTMs. The respondents explored pen-based interaction (Wacom tablet) and multi-touch interaction on a small and a large portable display (iPod Touch and iPad) in a simulated Pre-op setup where interaction was synchronized with a large display (see Figure 6). The user study was conducted with one physician at a time, with the study leader acting as radiologist, in control of the large display (which represented the projected screens used in the MDTMs). Two authentic patient cases (a liver and a pancreas case) were prepared in advance, including radiology images and text from the patient record (i.e., the radiology documentation from the Pre-op).
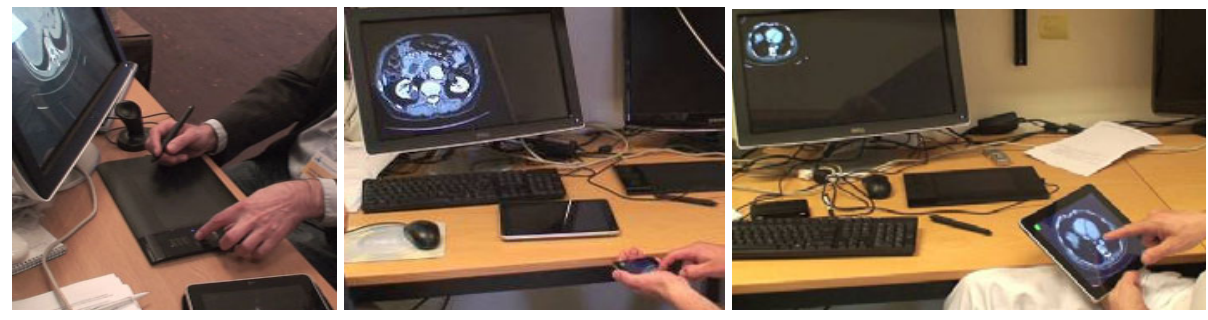

Fig. 6. Photographs from our user study, where participants explored the pen-based device (left), a small (middle) and a large (right) multi-touch display. The monitor represents the shared projection screen at the MDTMs.

We report on the results from individual sessions with eleven surgeons (one female) and one senior radiologist from the hospital. Seven of the surgeons were senior gastro surgeons specialised on a specific organ or intervention technique, four were consultants, four were gastro surgeons, one was a resident and one was a visiting gastro surgeon. The four consultants would typically lead the discussions, and the surgeons or the resident would present the case at the MDTMs. The radiologist was asked to respond to the study as if he was attending an MDTM where another radiologist demonstrated the material. The choice of focusing on surgeons is based on our field data showing that they are usually the ones asking more specific questions about the demonstrated imagery. We will broaden our studies to include more disciplines in the future. While the respondents lack experience in using ICT at these meetings, and access to technology is usually limited to PCs and medical devices, most of them were interested in exploring new technology that could support their 
work. Most of them had used or were familiar with mobile touch-screen devices, knew about iPads but had not used one or seen one in real life, and were not familiar with Wacom Tablets. The oldest respondent was known, among the surgeons, to be conservative about new technology. The respondents were 35-62 years old (avg. 42 years) and received no compensation. The sessions lasted 18-52 min (avg. $36 \mathrm{~min}$ ).

The study's objective was to identify and assess the potential of the implemented interfaces and technologies, and the type of functionality that would be useful at MDTMs. Questions from the study leader accompanied a pre-test questionnaire to collect initial attitudes. The respondents then explored the three interfaces (in randomized order) using think-aloud, combined with interview questions during the test, and a post-test questionnaire, where the usefulness of navigation, pointing and annotation in the different meeting situations, were respectively ranked on six-point Likert scales. Questionnaire data was not analysed statistically due to the number of respondents.

The respondents were asked to primarily consider the technology for Pre-op meetings, but the concluding discussion also concerned its applicability in other meetings or situations. The respondents were encouraged to comment and discuss with the study leader throughout the whole session. Sessions were recorded on video, transcribed and analysed in detail by categorising the data based on the current situation, potential risks, situations in which the devices could be used, and feedback on the interaction techniques and the input devices.

While individual sessions cannot fully simulate the multi-user interaction at the MDTMs, they were intended to allow us to gather focused feedback from each respondent without time constraints. The most senior participants at the MDTMs tend to dominate discussions given the formal experience-based hierarchies that dictate meeting roles. We thus balance our aim for a focused session and qualitative feedback from individuals with varying expertise and background, by having the interviewer take the role of the radiologist (controlling imagery from the workstation) with the participant simultaneously interacting using different devices. All respondents are well familiar with the MDTMs, and all senior surgeons and the radiologist attend every week. Two of the respondents also participated in the earlier study with laser pointers.

\section{Results}

The main target of the interaction in today's MDTMs is the radiology presentation and images. The radiologist is in control of the presentation and the other participants have to rely on other means of interacting with the images, for example when clarifications or more details are needed. In the pre-test discussion and questionnaire, almost all respondents emphasized the need for interaction with the images, especially pointing: "Some colleagues have laser pointers ... They use them to point ... Several approach and point in the projection" (resident F). However, some respondents stressed that Pre-ops are more suitable for interaction than Decision Meetings due to the large number of cases presented there. 


\subsection{Interaction Techniques: Pointing, Navigation and Annotation}

Of the three interaction techniques, the respondents were most excited about pointing, which could help resolve ambiguous references, avoid misunderstanding, and improve communication for local and remote users: "Pointing at a detail ... is very important" (senior surgeon U). Four physicians said that they today rely on verbal explanation using common terminology (e.g., the eight liver segments can be referenced by index), point with their finger towards the projected imagery, or walk up to the projection screens to point. Some respondents found this acceptable and not a problem that they had reflected on as they considered verbal references easy to understand for the radiologist: "You just say back a little ... scroll some" (senior surgeon B) and "I say that I want to see the blood vessel there" (senior surgeon A). The ineffectiveness of verbal references was pointed out by several respondents who found it time-consuming to explain to the radiologist which area they would like them to navigate to. They mentioned that it was an abstract way of pointing and could cause misunderstandings due to the difficulty of verbally directing the radiologist, and that participants may thus hesitate to ask for clarifications.

Precise and unambiguous pointing would also make the discussion easier to follow for surgeons in training. Senior physicians tend to have developed a more precise language and can therefore refer more accurately to specific parts of the images, while it is typically more difficult for junior surgeons to interpret and follow the discussion: "The experienced colleagues can use a completely different language, they are more precise than I can handle ... often they point, sort of sweeping ... but it's not really obvious what they point to" (resident F). The possibility for clearer pointing would, according to the resident, make it easier to follow and learn from the discussion. Thus, although experienced surgeons may be able to give accurate descriptions of areas of interest without pointing devices, their use of such devices might still be a great aid to less experienced surgeons.

Feedback on navigation primarily focused on image stack scrolling, while zooming and panning was emphasized for usability on the small display. Many respondents were not convinced about the need for navigation during MDTMs as they considered it the radiologist's job: "The radiologist presents the material very thoroughly and can see much better than I" (surgeon C).

Annotations seemed less important for the Decision Meeting discussions, but numerous comments indicate potential benefit in other situations. As for precise pointing, referencing would be clearer for, e.g., residents and junior surgeons, when lesions and relations to arteries can be highlighted. Basic functionality, which radiologists have access to today, such as marking and measurement tools, were frequently asked for by several respondents. Also, one surgeon commented that drawn annotations could be useful in specific cases that need more detailed discussion.

Some surgeons emphasized the ability to save annotations: "There is no point in marking temporarily - you'd want to save and continue later" (surgeon T) and "You'd mark the area that is unclear after the radiology presentation. The tumour often grows into a blood vessel ... you could actually mark it and refer to it here [while using the iPod]" (surgeon S). This would help the surgeons understand patient cases faster if they could not attend the Pre-op, or wanted to refresh their memory in preparation for an MDTM or a surgical procedure. Simple symbols like arrows, labels 
and numbers could complement the documentation, but also be useful for lectures and educational material. For a better overview of the annotations in radiology examinations (where there are multiple annotations in the image stack), an index of annotations, highlighting of annotated slices, and linked bookmarks to annotations were suggested.

\subsection{Private Interaction vs. Shared Focus}

Several respondents were excited to interact on their own high-quality display with easy-to-use controls. The large multi-touch device was appreciated for its large and clear display. Some respondents disliked the smaller device, while some thought that it was sufficient in size: "I can see well on this one too, the zoom is so powerful" (surgeon J). Some respondents expressed interest in exploring the imagery on their own and requesting control upon finding something they wanted to share: "You could work maybe a little in parallel, not having to disturb everyone” (senior surgeon B) and "The radiologist is presenting, and you have your iPhone to see what he is presenting ... then you see something important, and you can annotate it on your own [device]" (surgeon T). Still, it was generally considered that the radiologist should be in control of image manipulation on the shared display. If there was something respondents wanted to ask about or bring the group's attention to, they could navigate and annotate on their personal device and ask the radiologist for permission to show it on the shared display.

Several respondents were, however, concerned that users would only focus on their private screen - a disadvantage compared to the screen-less tablet: "The objective of the meeting is to have shared discussions ... if everyone is looking at their own [screen], then it isn't a meeting" (surgeon J), and "It would be bad for the group discussion [if you had your own]" (senior surgeon M). Also the radiologist commented on this: "I may loose their attention when I demonstrate details ... they might not even hear what I say — they would be into their own analysis ... it is better that everybody has the same focus".

\subsection{Applicability for Different Scenarios}

Many respondents pointed out that the evaluated tools would be much more valuable at Pre-ops as the discussions in these meetings are more focused, the groups are smaller, there are fewer patients to discuss and the meetings are not under time pressure, in contrast to Decision Meetings. One surgeon said: "There cannot be anything that disturbs the radiologist [at the Decision Meetings]. If you loose five minutes per patient, and have 19 patients ... That doesn't work" (surgeon T). Postops, on the other hand, are both less common (pancreas cases only) and less critical, as no decisions are taken. The respondents also commented on making annotations before the meeting, in order to save time during the meeting: "[It] can be good to mark which structure is which - this is actually truncus hiliacus ... and that can be done in advance ... it is also how much time it [annotating the images] will take from the meeting" (surgeon $\mathrm{J}$ ).

The benefits of clarity and accessibility were, in particular, highlighted by the less experienced surgeons. The resident said that less experienced surgeons would be able 
to follow the discussion better and would not need to disturb the more experienced surgeons if they can not follow what is referred to.

During the user studies, respondents also came to think of situations during the entire patient care pathway where the devices could be used. In team-based care, where multiple physicians share the responsibility for patients, it is important to be able to hand over patient information such that another physician can easily catch up on a case and quickly be able to make decisions. Decision Meeting protocols should accompany the imagery and examinations on to the Pre-op, where the surgical plan is annotated on the images. The plan could then be used when preparing the surgical procedure, especially if the surgeon in charge of the operation was not present at the Pre-op: "The image information is very important ... today it [the surgical planning] is based on the Pre-op and that the surgeon is present to see and get a mental image for making a strategy to remember" (surgeon J). The plan could be shown both to the patient and to the team in the operating room, and in the end ideally all the material could be accessible for presentation at a Post-op or follow-up meeting: "It would be elegant if s/he [the surgeon] could display the images: 'This is what we planned', 'This is what we did ... but when we physically got there ... this structure was there, and we decided to go here' [simultaneously pointing in the image]" (resident F). Some respondents found other situations more suitable than the MDTMs: "I think this is fantastic [while using the iPod] ... then you can save, store and work with it on your own computer before the operation. ... The radiologists could annotate important things and I'd look at it on my computer later on. That would be more useful than if I annotate" (surgeon T).

\subsection{Interaction Technology: Multi-touch, Pens, Display, Size, Precision and Portability}

Although respondents only used the three devices for about ten minutes each, most were able to give feedback on usability and ergonomics. On the other hand, the respondents were not always consistent in their preferences, as they might have preferred different devices depending on situation and purpose.

The same user interface and functionality was implemented for the two multi-touch devices. The smaller device was appreciated for its portable size, but at the same time several respondents found the display too small for efficient use. Numerous respondents, when interacting with it, only looked at the large, shared display. One respondent even stated that the small display was not needed, as it was sufficient to control the content on the shared display with multi-touch input. The zoom function of the multi-touch devices was much appreciated, as well as the responsive interface and display quality. The large device inherits most of the perceived positive properties from the smaller, but its size accounted for better precision and overview, and was therefore generally more appreciated.

Initially, the pen-based tablet was perceived as complicated to use since the functionality relied on two buttons, a scroll wheel and the pen: "I can tell you directly that this seems awkward" (senior surgeon U). On the other hand, when the respondents had used it for a few minutes, seven of them appreciated the device primarily for its high precision when pointing and drawing, and due to the fact that the large display must be viewed (which during a meeting would make participants 
focus on the same display): "Once you get used to it, it seems quite user-friendly, in my opinion ... this is good enough for drawing” (surgeon J).

Two respondents did not prioritize drawing precision: "Precise sketching is not that important because I think sketches and annotations are only important when referring to the operating surgeon that you haven't missed anything, that you haven't missed any point that was discussed" (surgeon S) and "The sketches would be quick, you wouldn't have to be very detailed [while using the Wacom]" (surgeon J). Three respondents were disappointed that they were not able to also use a stylus on the touch-screen devices. Two respondents believed that a high-precision device would probably be more relevant for the radiologist and the chair of the meeting, since other participants would probably not need the same level of precision. Thus, according to those participants, not everyone in the meeting needs to be able to interact with the medical imagery through networked devices.

Seven respondents were impressed by the interface and portability of the smaller multi-touch device while the larger was considered, by four respondents, too heavy $(680 \mathrm{~g})$ and large $(1.34 \times 19 \times 24.3 \mathrm{~cm})$ to carry around. Several respondents described ways in which they could be more effective if they had access to portable technology: benefits during individual preparation (e.g., at home, the evening before surgery) and for patient consultation. One of the surgeons said "I would look briefly right before surgery" (senior surgeon B), and another one said “... in a hospital round ... [to patient] here is the problem, and we will solve it by removing this part” (Surgeon S).

\subsection{Suggested Improvements, Additional Functionality and Potential Risks}

It should be stressed that most respondents believed that a limited set of basic and easy-to-use tools (for pointing, navigation and annotation) was sufficient. However, the respondents did also suggest additional functionality that could support other situations in their work. As all respondents, except one, were surgeons, they did not have access to a radiologist's set of tools, and some of them mentioned features such as copying or overlaying annotations over an image sequence, comparing marked-out lesions over different image slices or examinations, and splitting the screen into two or four viewports.

Text annotations were frequently suggested as support for, and clarification of, discussed findings in the images. One surgeon said "Put the number one here, and write what we plan to do" (resident F). These text annotations could be combined with standardized options, accessible through, e.g., a drop-down menu, for simplicity and consistency. Some respondents even speculated about replacing the Pre-op meeting protocol with a set of text-annotated images as the predefined options could mandate a standard for the required information. Annotations from the meetings should of course be linked to the medical record, and physicians should at any time be able to access them.

Due to the way pointing was implemented on the devices (a circle with a size relative to the different display sizes) several respondents wanted a more precise pointing tool, such as an arrow.

While almost all respondents were enthusiastic about the possibilities of using interaction tools, a few concerns were raised about a multi-user system. Several surgeons commented that the radiologists are currently in control of demonstrating the 
images and that each speciality should do what they are best at. Most respondents were concerned about "chaos" if multiple people tried to interact simultaneously with the radiology images. Several respondents suggested that this would probably be resolved through social protocols: "You'd have to take turns, just like when we speak" (surgeon J). Some respondents suggested that the radiologist should delegate control when needed. Another suggestion was that maybe not all participants should have devices, but only a few senior persons.

\section{Discussion}

Our initial studies, as well as field studies by other researchers (e.g. [7, 8]), show that surgeons often point to certain areas in medical images during MDTMs. The results from these earlier studies, and interview results from this study, suggest that using gestures far away from the projected images is neither sufficiently clear nor precise. Technical support for pointing and annotating seem to be able to alleviate such problems. Although not tested for statistical significance, our qualitative data gives strong indications for the potential of such interaction technology.

Of the three interaction techniques that we evaluated, pointing was considered most important. Our qualitative data highlighted the potential for precise pointing with digital tools that are synchronized across multiple displays, as it may save time in the discussion, include remote sites and make references more explicit for less experienced participants. Varying level of pointing precision should be supported on all devices, as a physician might want to refer to both small details and larger areas in an image. This would benefit discussions by minimizing ambiguous referencing. Annotation can, on the other hand, be accomplished with lower precision. It is not important to, for instance, exactly outline a lesion, or draw the exact path for incision during pre-operative planning. It is sufficient to highlight the area in which a lesion is located, or sketch the hypothetical incision, as the exact incision can only be determined during surgery.

Annotations could help clarify the discussion further, but were considered perhaps even more interesting when saved with the imagery for use in other situations. As anticipated, these techniques were confirmed to be most useful for Pre-ops, whereas the system should allow the data to be reused along the patient care pathway, such as for individual preparation before surgery or in Post-ops. Annotations made during the meetings, both in the form of sketches or, as several physicians suggested, text, should be saved for future use. As the physicians' work is team-based and several physicians access the patient records during the patient care pathway, annotations would help information handovers. Radiology images augmented with additional information would make it easier and faster to understand what was discussed in a meeting, overview the most important images and findings, and review proposed surgical strategies.

While most study participants felt comfortable with the radiologist navigating the material, implicit navigation on the devices is obviously necessary in the user interface to support interaction with areas of interest.

Concerns were raised about issues that, for example, could arise with personal displays that distract from the discussion, or if multiple users attempted simultaneous 
interaction. Thus, social protocols and technical policies that control the use need to be explored and evaluated before implementation.

Kane and Luz also discuss new technology's effect on group dynamics [8]. Often, participants already have a pre-defined role during the MDTMs, which would be affected by the introduction of tools that delegate control to other participants, an issue mentioned by several of our study participants. There was, e.g., concern that senior surgeons might start dominating MDTMs with less experienced radiologists. Surgeons and radiologists in our study, however, thought that communication protocols for turn taking would develop in the same way as when they are talking.

The three devices had their respective advantages and disadvantages, such as the pen's precision vs. the multi-touch interaction that was considered more intuitive, the ergonomic advantage of a large display size vs. portability, or the existence of a display vs. the potential risk for distraction from the shared screen. Whether, for example, personal navigation should be implemented during MDTMs, or if only a shared view should be used was also an issue identified by Wallace et al. [23], and our next step is to explore multi-user interaction during real MDTMs to evaluate how this technology affects group dynamics and to assess the importance of personal interaction. We emphasize that it is not the comparison of the devices themselves that is valuable in this context, but the qualities the study participants appreciated or found relevant.

Interestingly, several surgeons spontaneously suggested additional activities and scenarios in which the technology could be used, as there are many activities besides MDTMs in the patient care pathway. Surgery planning and preparation (e.g., the morning before the surgery) and patient consultation, were examples of other scenarios where participants thought the interactive devices could be useful. This is in accordance with previous research [4], which shows that cooperative and participatory design activities made them reflect on their own work and processes.

Physicians are also becoming increasingly mobile and will need access to updated, relevant patient information in different situations. Mobile devices could support a number of such situations, from hallway discussions to post-op reviews of complete cases. Personal navigation in a surgeon's office, for example, could be useful to understand anatomy and lesions for a specific case. Before performing surgery, the surgeon sometimes examines a 3D reconstruction of the volume. It is, in general, important for the surgeons to follow anatomical features (such as blood vessels, organs and lesions), to see how they intersect, for example. This is a functionality they all need, independent of activity (MDTMs, personal navigation or hallway discussions).

\section{Conclusions and Future Work}

We have developed and evaluated interaction technology for supporting emerging needs in multi-disciplinary collaboration for specialized medical care. Interviews, observations and a qualitative user study with surgeons and radiologists from different disciplines and varying levels of expertise, provided us with interesting insights for the next generation of digital tools for MDTMs. Unsurprisingly; the reactions were 
overall positive, as our developed system clearly demonstrated the potential benefits of new interactive capabilities.

Our interaction techniques (pointing, navigation and annotation) for multi-user interaction were implemented on two multi-touch devices (small and large) and a penbased tablet. The use of laser pointers that have been observed by several researchers [7, 15] does not support the typical multi-site video-mediated MDTMs. Digital tools do, in contrast, address the problems caused by, e.g., laser pointers that are not visible to all collaborating participants, without requiring additional hardware installation (e.g., cameras for laser pointer tracking [17]). While the proposed technology for augmenting the meetings is advanced, we emphasize the importance of the perceived simplicity of the interaction techniques. We believe that three classes of devices are relevant for further exploration at MDTMs: Simple pointing tools (e.g., multiple wireless mice or trackpads), portable devices (e.g., mobile devices with large touch screens) and larger wireless devices with high-resolution displays that support both stylus (precision) and touch input (e.g., Tablet PCs).

We build on previous work and field studies, by introducing technology and strategies for interacting with information presented during MDTMs and provide a set of design guidelines, the importance of which were implicitly confirmed in the study, that we hope will serve as inspiration for future MDTM systems. Our results show that more precise referencing could augment the expressiveness of participants, improve the communication with the other participants (including the radiologist), help less experienced participants follow the discussion, and bridge the gap to remote experts.

By introducing distributed interaction along with proper social protocols and system policies, its potential for improved collaboration, understanding and discussion could lead to safer and faster medical decisions, with a significant impact for modern healthcare.

This study gathered insights during a controlled simulated team meeting in preparation for follow-up studies during real Pre-operative meetings. It allowed us to collect valuable unbiased, undisturbed and undistracted feedback through a series of individual sessions. Based on the results from this work, we are now refining the techniques and the setup, and plan to run experiments with the next generation of our prototypes in collaborative multi-user meetings.

\section{References}

1. Bier, E.A., Freeman, S.: MMM: a user interface architecture for shared editors on a single screen. In: Proc. UIST 1991, pp. 79-86 (1991)

2. Eng, J., Leal, J. P., Shu, W., Yang, G.L.: Collaboration System for Radiology Workstations. Radiographics 22, e5 (September 2002); Published online August 21

3. Flanders, A.E., Wiggins III, R.H., Gozum, M.E.: Handheld Computers in Radiology. Radiographics 23, 1035-1047 (2003)

4. Frykholm, O., Lantz, A., Groth, K., Walldius, Å.: Medicine Meets Engineering in Cooperative Design of Collaborative Decision-supportive System. In: Proc. CBMS 2010 (2010) 
5. Groth, K., Frykholm, O.: Efficiency in Treatment Discussions: A Field Study of Time Related Aspects in Multi-Disciplinary Team Meetings. In: Proc. CBMS 2009, pp. 1-8 (2009)

6. Groth, K., Olin, K., Gran, O., Permert, J.: The role of technology in video-mediated consensus meetings. Journal of Telemedicine and e-Health 14(7), 349-353 (2008)

7. Kane, B., Luz, S.: Multidisciplinary Medical Team Meetings: An Analysis of Collaborative Working with Special Attention to Timing and Teleconferencing. Journal of CSCW 15, 501-535 (2006)

8. Kane, B., Luz, S.: Achieving Diagnoses by Consensus. Journal of CSCW 18, 357-392 (2009)

9. Kane, B., Luz, S., O’Brian, D.S., McDermott, R.: Multidisciplinary team meetings and their impact on workflow in radiology and pathology departments. BMC Medicine 5, 15 (2007)

10. Lee, S.-K., Peng, C.-H., Wen, C.-H., Huang, S.-K., Jiang, W.-Z.: Consulting with Radiologists outside the Hospital by Using Java. Radiographics 19, 1069-1075 (1999)

11. Li, J., Mansfield, T., Hansen, S.: Supporting Enhanced Collaboration in Distributed Multidisciplinary Care Team Meetings. In: Proc. CBMS 2008, pp. $482-487$ (2008)

12. Morris, M. R., Huang, A., Paepcke, A., Winograd, T.: Cooperative gestures: multi-user gestural interactions for co-located groupware. In: Proc. CHI 2006, pp. 1201-1210 (2006)

13. Myers, B.A., Stiel, H., Gargiulo, R.: Collaboration using multiple PDAs connected to a PC. In: Proc. CSCW 1998, pp. 285-294 (1998)

14. Myers, B.A.: Using Hand-Held Devices and PCs Together. Communications of the ACM 44(11), 34-41 (2001)

15. Måseide, P.: The deep play of medicine: Discursive and collaborative processing of evidence in medical problem solving. Communication \& Medicine 3(1), 43-54 (2006)

16. Paek, T., Agrawala, M., Basu, S., Drucker, S., Kristjansson, T., Logan, R., Toyama, K., Wilson, A.: Toward universal mobile interaction for shared displays. In: Proc. CSCW 2004, pp. 266-269 (2004)

17. Olsen, D.R., Nielsen, T.: Laser pointer interaction. In: Proc. CHI 2001, pp. 17-22 (2001)

18. Ratib, O., Michael McCoy, J., Ric McGill, D., Li, M., Brown, A.: Use of Personal Digital Assistants for Retrieval of Medical Images and Data on High-Resolution Flat Panel Displays. Radiographics 23, 267-272 (2003)

19. Rekimoto, J.: A multiple device approach for supporting whiteboard-based interactions. In: Proc. CHI 1998, pp. 344-351 (1998)

20. Ruhstaller, T., Roe, H., Thürlimann, B., Nicoll, J.J.: The multidisciplinary meeting: An indispensable aid to communication between different specialities. European Journal of Cancer 42(15), 2459-2462 (2006)

21. Stewart, J., Bederson, B.B., Druin, A.: Single display groupware: a model for co-present collaboration. In: Proc. CHI 1999, pp. 286-293 (1999)

22. Tse, E., Histon, J., Scott, S.D., Greenberg, S.: Avoiding interference: how people use spatial separation and partitioning in SDG workspaces. In: Proc. CSCW 2004, pp. $252-$ 261 (2004)

23. Wallace, J.R., Scott, S.D., Stutz, T., Enns, T., Inkpen, K.: Investigating teamwork and taskwork in single- and multi-display groupware systems. Personal and Ubiquitous Computing 13(8), 569-581 (2009) 